Revisión y/o Actualización de Literatura

\title{
Carga mental en profesores y consecuencias en su salud: una revisión integrativa
}

\author{
Carga mental em professores e suas consequências para saúde: uma \\ revisão integrativa
}

\section{Mental workload in faculty and consequences in their bealth: an integrative review}

\author{
Yanni Lonnette González-Palacios ${ }^{a}$ (D), Paula Andrea Ceballos-Vásquez ${ }^{a}$ (D), Flérida Rivera-Rojas ${ }^{a}$ (D) \\ ${ }^{a}$ Facultad de Ciencias de la Salud, Universidad Católica del Maule - UCM, Talca, Chile.
}

Cómo citar: González-Palacios, Y. L., Ceballos-Vásquez, P. A., \& Rivera-Rojas, F. (2021). Carga mental en profesores y consecuencias en su salud: una revisión integrativa. Cadernos Brasileiros de Terapia

Ocupacional, 29, e2808. https://doi.org/10.1590/2526-8910.ctoAR2123

\begin{abstract}
$\underline{\text { Resumen }}$
Introducción: La literatura en cuanto al contexto laboral de los profesores ha documentado las condiciones de trabajo y sus efectos en la salud, sin embargo, existe escasa evidencia de los efectos de la Carga Mental de Trabajo (CMT) en este grupo, pese a tratarse de un riesgo psicosocial que se construye a partir de factores propios del quehacer docente. Objetivo: Explorar la literatura respecto a la CMT percibida por profesores y su relación con condiciones de trabajo y efectos en su salud. Método: Se realizó revisión integrativa en bases de datos Web of Science, CINAHL Complete, PubMed y Scielo utilizando descriptores en inglés y español considerándose como criterios de inclusión estudios cuantitativos, cualitativos y mixtos; estudios publicados desde 2013 al 2019. Además, se realizó búsqueda manual, rescatando un estudio de relevancia para el abordaje de la temática. Los estudios fueron sometidos a evaluación según niveles de evidencia desarrollados por el Oxford Centre forEvidence-Based Medicine. Resultados: Se localizaron 20 artículos y se formularon categorías de análisis: condiciones laborales de profesores, efectos del trabajo en la salud, carga mental percibida por los mismos, además de establecerse sugerencias para la salud ocupacional. Conclusión: Las características del contexto docente incluyen factores propios de la CMT, entre ellos la intensidad laboral y tiempos de trabajo extenuante, ruido, alta cantidad de estudiantes, mala calidad de relaciones interpersonales, inestabilidad laboral y falta de recursos.
\end{abstract}

Palabras clave: Riesgos Laborales, Docentes, Salud Laboral.

\section{$\underline{\text { Resumo }}$}

Introduçáo: A literatura referente ao contexto de trabalho dos professores tem documentado as condiçóes de trabalho e seus efeitos na saúde, porém existem poucas evidências dos efeitos citado. 
da Carga Mental de Trabalho (CMT) neste grupo, embora a CMT possa ser um risco psicossocial que se constrói por meio dos fatores do trabalho docente. Objetivo: Explorar na literatura a carga mental percebida pelos professores, sua relaçáo com as condiçôes de trabalho e seus efeitos na saúde. Método: Realizou-se revisão integrativa nas bases de dados Web of Science, CINAHL complete, Pubmed e Scielo, utilizando os descritores em inglês e espanhol. Os critérios de inclusăo foram: estudos quantitativos, qualitativos e mistos, publicados a partir de 2013 até 2019. Além disso, foi realizado uma busca manual resgatando um estudo de relevância que abordam a temática. Os estudos foram avaliados de acordo com os níveis de evidência desenvolvidos pelo Oxford Center for Evidence-Based Medicine. Resultados: Incluíram-se 20 artigos que foram organizados em categorias de análise: condiçóes laborais dos professores, efeitos do trabalho na saúde, carga mental percebida e sugestôes para a saúde ocupacional. Conclusáo: As características do contexto docente incluem fatores específicos da CMT, incluindo a intensidade laboral, tempo de trabalho deficiente, ruído, elevado número de estudantes, relaçóes interpessoais de baixa qualidade, falta de estabilidade no trabalho e falta de recursos.

Palavras-chave: Riscos Ocupacionais, Docentes, Saúde do Trabalhador.

\section{$\underline{\text { Abstract }}$}

Introduction: The literature regarding the teachers' work context has documented working conditions and their effects on health. However, there is limited evidence of the Mental Workload (MW) effects in this group, despite being a psychosocial risk that is constructed from typical factors of teaching work. Objective: Literature about mental workload perceived by professors and their relation with work conditions and the effects on their health were explored. Method: An integrative review was realized in databases Web of Science, CINAHL Complete, PubMed, and Scielo using descriptors in English and Spanish. The inclusion criteria were quantitative, qualitative, and mixed studies published between 2013 and 2019. In addition, a manual search was made obtaining relevant one study for the approach of the subject. The studies were evaluated according to levels of evidence developed by the Oxford Center for Evidence-Based Medicine. Results: 20 articles were selected and divided into analytical categories: work conditions of professors, work effect on health, mental workload perceived by them, in addition to establishing suggestions for occupational health. Conclusion: The characteristics of the work environment include factors specific to mental workload, including high work intensity and strenuous work times, noise, a high number of students, bad interpersonal relationships, work instability, and lack of resources.

Keywords: Occupational Risks, Faculty, Occupational Health.

\section{Introducción}

En salud ocupacional, se tiene presente que diferentes factores pueden generar efectos en las personas, acorde a lo mencionado por la Organización Mundial de la Salud (OMS), quien reconoce que el bienestar de los trabajadores no sólo se ve condicionada por peligros en el lugar de trabajo, sino también por factores sociales e individuales, y por el acceso a los servicios (Organización Mundial de la Salud, 2015). Los profesionales sanitarios en su rol en la promoción de conductas saludables, prevención de enfermedades y riesgos para la salud, han 
visibilizado la necesidad de construir entornos laborales saludables (Ceballos et al., 2016), estableciendo diferentes directrices en este ámbito para el desempeño tanto de enfermeros, médicos, kinesiólogos, nutricionistas, terapeutas ocupacionales, entre otros profesionales, evidenciando que deben enfocar sus tareas en la prevención de enfermedades, accidentes de trabajo y promoción de salud ocupacional, debiendo responder al cambio paradigmático del mundo laboral, que ha sufrido modificaciones a raíz de avances en tecnología, políticas públicas y legislaciones en el área (Boada-Grau \& Ficapal-Cusí, 2012; Ceballos, 2015; Murillo et al., 2020). Es por esto que se han estudiado diferentes factores, involucrados en los efectos del contexto laboral sobre la salud.

La Carga mental de trabajo (CMT) corresponde a uno de ellos, y es utilizada para referirse a las tensiones que provocan en el trabajador las exigencias laborales, así como la relación que se produce entre las exigencias del trabajo y las capacidades mentales o recursos propios de la persona para afrontarlas, y se relaciona con tareas que implican procesos cognitivos y aspectos afectivos del individuo (Ferreira \& Ferreira, 2014, p. 48). La CMT es un fenómeno multidimensional, la cual debe ser considerada durante el diseño de cualquier tarea de trabajo y, según la norma UNE-ISO 10075, es el resultado de la combinación de diferentes factores como lo son las características personales, de la tarea y de la situación, y cuyo desbalance puede generar en el trabajador la percepción de riesgos psicosociales (Díaz et al., 2012). Estos últimos se dan principalmente en contextos laborales del área de servicios como transportes, retail y educación.

La educación es un área de trabajo para múltiples personas, y se caracteriza por ser un entorno amplio y diverso en el que se reconocen diversos factores psicosociales. Es más, el ejercicio docente ha sido considerado como una de las profesiones más propensas a percepción de burnout y malestar emocional entre sus trabajadores (Ilaja \& Reyes, 2016), incluso generando disconformidad con las condiciones laborales en gran parte de ellos (ZamoraDíaz et al., 2017). En el ámbito de la educación primaria y secundaria, se han realizado numerosos trabajos para caracterizar el entorno y condiciones de trabajo de los docentes, y cuyos resultados han evidenciado actividades con alta demanda física, mental y emocional, condiciones laborales insuficientes y un ritmo alto de trabajo (Boström et al., 2019; Silva \& Pinheiro, 2017). Debido a esto es que surge el interés de determinar otros factores presentes en la labor de los docentes que pudieran tener efectos en su salud. Así, es que el objetivo de esta revisión es explorar lo que la literatura internacional ha abordado sobre la carga mental de trabajo percibida por profesores y su relación con las condiciones de trabajo y los efectos en la salud de éstos.

\section{Método}

Se desarrolló una revisión integrativa de la literatura a nivel internacional, para identificar los datos referentes a la carga mental de trabajo percibida por los profesores de educación primaria y secundaria, junto con las condiciones laborales y las repercusiones en su salud.

La revisión se llevó a cabo bajo la metodología de Souza et al. (2010), la que consta de seis pasos para la construcción de la revisión integradora de la literatura: 1) Preparación de la pregunta guía para la elaboración de la revisión integradora, 2) búsqueda de la literatura, 3) recolección de la información que se extraería de los estudios seleccionados, 4) análisis crítico de los estudios incluidos en la revisión integradora, 5) discusión de los resultados y 6) presentación de la revisión integradora. 
El manuscrito emerge con motivo de dar respuesta a la pregunta norteadora: ¿Existe relación entre la carga mental percibida por los profesores de educación primaria y secundaria, las condiciones laborales y los efectos en su salud?, la cual se formuló a partir de una adaptación de la estrategia PICOT (Peñaherrera \& Soria, 2015) para usar en esta revisión.

Se consultaron las bases de datos Web of Science, CINAHL Complete, PubMed y Scielo utilizando los descriptores "Carga mental", "Profesores", "Docentes" y en inglés "Mental workload", "Teachers" y "Schoolteachers". Se establecieron los siguientes criterios de inclusión: a) Estudios cuantitativos, cualitativos y mixtos, excluyéndose en primera instancia resúmenes, extractos y artículos de pago, b) Antigüedad desde el año 2013-2019. Posteriormente se realizó una búsqueda manual con los mismos descriptores, y se rescató un estudio, por su relevancia para la temática.

Para la recolección de los datos se utilizó un instrumento de elaboración propia, compuesto por ocho ítems, que aborda las siguientes variables: revista, indexación, año, lugar de ejecución, método, muestra, principales resultados y nivel de evidencia. Ésta última variable fue clasificada según los niveles de evidencia desarrollados por el Oxford Centre for Evidence-Based Medicine (OCEBM) (Oxford Centre for Evidence-Based Medicine, 2011). La sistematización de la búsqueda bibliográfica se resume en la Figura 1.

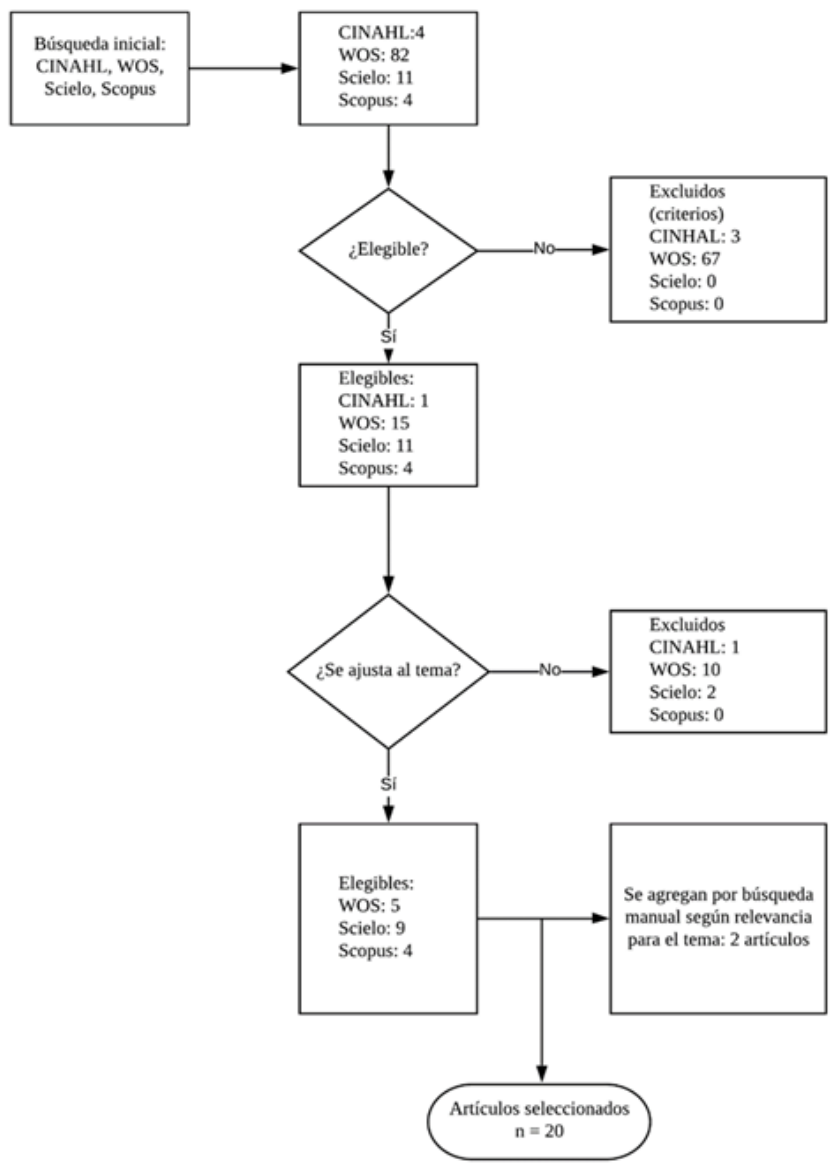

Figura 1. Flujograma de la revisión de la literatura. Fuente: Elaboración propia. 


\section{Resultados}

La búsqueda bibliográfica arrojó 20 artículos en total, de los cuales el 85\% son de abordaje cuantitativo, y el resto son de abordaje cualitativo y mixto. Se destaca que el $75 \%$ de los estudios fueron realizados en países latinoamericanos, un 15\% se desarrollaron en Europa, y 10\% en Norteamérica y Asia. Respecto a la indexación, se indica que un 50\% de los artículos están indexados en Scielo, el 25\% están indexados en WOS, y el resto de los artículos se localizaron en Scopus y Latindex. A continuación, en la Tabla 1 se describen los artículos seleccionados para analizar dentro de la revisión bibliográfica y su clasificación según nivel de evidencia (Oxford Centre for Evidence-Based Medicine, 2011).

Tabla 1. Descripción de artículos seleccionados en la revisión bibliográfica.

\begin{tabular}{|c|c|c|c|c|}
\hline Código & $\begin{array}{c}\text { Revista/ } \\
\text { Indexación, año y } \\
\text { lugar de ejecución }\end{array}$ & Método y muestra & Principales resultados & $\begin{array}{l}\text { Nivel de } \\
\text { evidencia }\end{array}$ \\
\hline E.1 & $\begin{array}{l}\text { Trabalho, } \\
\text { Educação e Saúde/ } \\
\text { Scielo. 2016. Brasil } \\
\text { (Guerreiro et al., } \\
\text { 2016) }\end{array}$ & $\begin{array}{l}\text { Cuantitativo, } \\
\text { transversal, muestra } \\
978 \text { profesores. }\end{array}$ & $\begin{array}{l}\text { Los docentes refieren factores negativos que } \\
\text { condicionan su trabajo: infraestructura del } \\
\text { lugar de trabajo, remuneración y el número } \\
\text { de estudiantes por sala. Los factores positivos } \\
\text { son las relaciones con alumnos, colegas y } \\
\text { directores, y motivación y oportunidad para } \\
\text { expresar opiniones. }\end{array}$ & 3 \\
\hline E.2 & $\begin{array}{l}\text { Cadernos } \\
\text { Brasileiros de } \\
\text { Terapia } \\
\text { Ocupacional/ } \\
\text { Scopus. 2017. } \\
\text { Brasil (Silva \& } \\
\text { Pinheiro, 2017) }\end{array}$ & $\begin{array}{l}\text { Cuantitativo, } \\
\text { descriptivo, } \\
\text { exploratorio, } \\
\text { muestra } 110 \\
\text { profesores }\end{array}$ & $\begin{array}{l}\text { Actividades burocráticas representan mayor } \\
\text { demanda física, tales como corrección de } \\
\text { materiales y actividades en grupo con } \\
\text { estudiantes, las cuales se pueden traducir en } \\
\text { dolor de miembros superiores e inferiores, } \\
\text { cabeza y columna. En cuanto a las demandas } \\
\text { mentales, la principal causa es el desinterés de } \\
\text { alumnos y padres, lo cual se pueden traducir } \\
\text { en síntomas emocionales como irritabilidad, } \\
\text { desánimo e insomnio, estrés, entre otros. }\end{array}$ & 4 \\
\hline E.3 & $\begin{array}{l}\text { International } \\
\text { journal on working } \\
\text { conditions/ } \\
\text { Latindex. 2017. } \\
\text { Portugal (Sousa \& } \\
\text { Barros, 2017) } \\
\end{array}$ & $\begin{array}{c}\text { Metodología mixta, } \\
\text { participaron } 89 \\
\text { profesores y } 9 \\
\text { escuelas de distrito. }\end{array}$ & $\begin{array}{l}\text { Existen alta exposición a tiempos de trabajo, } \\
\text { exigencias emocionales y mala calidad de las } \\
\text { relaciones sociales en el trabajo, provocando } \\
\text { situaciones de agotamiento y fatiga } \\
\text { generalizada que condicionan el ejercicio } \\
\text { saludable de sus actividades de trabajo. }\end{array}$ & 3 \\
\hline E. 4 & $\begin{array}{l}\text { Journal of Work } \\
\text { and Organizational } \\
\text { Psychology/ Web } \\
\text { of Science. 2015. } \\
\text { Brasil (Carlotto \& } \\
\text { Câmara, 2015) }\end{array}$ & $\begin{array}{l}\text { Estudio cuantitativo, } \\
\text { transversal, muestra } \\
679 \text { profesores de } 37 \\
\text { escuelas. }\end{array}$ & $\begin{array}{c}34,8 \% \text { de los profesores reportaron } \\
\text { trastornos mentales comunes. Algunas } \\
\text { variables psicosociales como ambigüedad del } \\
\text { rol, sobrecarga, bajo nivel de apoyo social y } \\
\text { bajo percepción de autoeficacia estuvieron } \\
\text { asociadas de manera significativa con } \\
\text { aumento en la probabilidad para trastornos } \\
\text { mentales comunes. }\end{array}$ & 3 \\
\hline E.5 & $\begin{array}{c}\text { Avances en } \\
\text { Enfermería/ Scielo. } \\
\text { 2013. Colombia } \\
\text { (Castro \& Muñoz, } \\
\text { 2013) }\end{array}$ & $\begin{array}{c}\text { Cuantitativo, } \\
\text { descriptivo } \\
\text { transversal, muestra } \\
\text { de } 230 \text { docentes. }\end{array}$ & $\begin{array}{l}\text { El 51,7\% de los docentes realizaba extensión } \\
\text { del trabajo, } 37,8 \% \text { reportó presencia de } \\
\text { ruido en aula. Las principales causas de } \\
\text { consulta médica en los docentes eran estrés, } \\
\text { un } 40,9 \% \text {, problemas vasculares y várices en } \\
\text { miembros inferiores con } 29,1 \% \text {, colon } \\
\text { irritable } 28,3 \% \text { y disfonías o afonías } 27,8 \% \text {. }\end{array}$ & 3 \\
\hline
\end{tabular}


Tabla 1. Continuación...

\begin{tabular}{|c|c|c|c|c|}
\hline Código & $\begin{array}{c}\text { Revistal } \\
\text { Indexación, año y } \\
\text { lugar de ejecución }\end{array}$ & Método y muestra & Principales resultados & $\begin{array}{l}\text { Nivel de } \\
\text { evidencia }\end{array}$ \\
\hline E.6 & $\begin{array}{l}\text { The Scientific World } \\
\text { Journal/ Scopus. } \\
\text { 2015. Brasil (Cezar- } \\
\text { Vaz et al., 2015) }\end{array}$ & $\begin{array}{l}\text { Cuantitativo, } \\
\text { exploratorio y } \\
\text { descriptivo. } \\
\text { Muestra } 37 \\
\text { profesores. }\end{array}$ & $\begin{array}{l}\text { Las condiciones de trabajo estresantes están } \\
\text { relacionadas con salario inadecuado, excesivo } \\
\text { número de actividades y tener que llevar trabajo } \\
\text { a casa. Las consecuencias evidenciadas son } \\
\text { ansiedad, estrés y desórdenes del dormir. Los } \\
\text { docentes reportaron que una buena relación } \\
\text { entre colegas promueve bienestar en el trabajo. }\end{array}$ & 4 \\
\hline E.7 & $\begin{array}{l}\text { Sao Paulo Medical } \\
\text { Journal/ Web of } \\
\text { Science. } 2015 . \\
\text { Brasil } \\
\text { (Baldacara et al., } \\
\text { 2015) }\end{array}$ & $\begin{array}{l}\text { Cuantitativo, } \\
\text { observacional, } \\
\text { transversal } \\
110 \text { profesores } \\
\text { municipales. }\end{array}$ & $\begin{array}{l}\text { El } 45 \% \text { de los profesores presentan síntomas } \\
\text { suficientes para considerar el diagnóstico de } \\
\text { trastorno mental y necesidad de tratamiento. }\end{array}$ & 4 \\
\hline E. 8 & $\begin{array}{c}\text { Revista Clínica } \\
\text { Contemporánea/ } \\
\text { Latindex. } 2018 . \\
\text { España (Guerrero- } \\
\text { Barona et al., 2018) }\end{array}$ & $\begin{array}{l}\text { Cuantitativo, } \\
\text { transversal, } \\
\text { correlacional }\end{array}$ & $\begin{array}{l}\text { Destacaron como fuentes de estrés la indisciplina, } \\
\text { la falta de motivación de los alumnos y la escasa } \\
\text { colaboración de las familias son las principales } \\
\text { fuentes de estrés. Quienes perciben altos niveles } \\
\text { de estrés presentan peor salud mental, } 36.3 \% \\
\text { muestra riesgo de trastorno psiquiátrico. Se } \\
\text { demostró una relación significativa entre el nivel } \\
\text { de estrés, los factores de riesgo psicosociales y la } \\
\text { salud mental. Además, se destaca que la } \\
\text { satisfacción laboral y el nivel de compromiso o } \\
\text { implicación predicen la salud mental. }\end{array}$ & 3 \\
\hline E.9 & $\begin{array}{l}\text { Ciencia y trabajo/ } \\
\text { Scielo. 2018. Chile } \\
\text { (Mora Pino et al., } \\
\text { 2018) }\end{array}$ & $\begin{array}{l}\text { Cuantitativo, } \\
\text { descriptivo, } \\
\text { transversal, muestra } \\
70 \text { profesores de } \\
\text { educación básica } \\
\text { municipal. }\end{array}$ & $\begin{array}{l}\text { En relación a la disfonía ocupacional, existen } \\
\text { factores personales y laborales-ambientales que } \\
\text { repercuten en su presentación. Dentro de los } \\
\text { personales destacan: hidratación insuficiente, } \\
\text { elevar intensidad de la voz, gritar y carraspear, } \\
\text { alto consumo de irritantes laríngeos. En cuanto } \\
\text { al contexto ocupacional se encontró que: } 82 \% \\
\text { de encuestados ocupa la voz por más de } 31 \text { hrs. } \\
\text { semanales, alta cantidad de alumnos, presencia } \\
\text { de corrientes de aire y cambios de temperatura } \\
\text { en sala de clases; exposición a ruido ambiental. } \\
\text { Falta de capacitación a los profesores sobre el } \\
\text { uso adecuado de la voz. }\end{array}$ & 4 \\
\hline E.10 & $\begin{array}{c}\text { International journal } \\
\text { of occupational } \\
\text { medicine and } \\
\text { environmental } \\
\text { health/ Web of } \\
\text { Science. } 2013 . \\
\text { Alemania (Seibt et al., } \\
\text { 2013) }\end{array}$ & $\begin{array}{l}\text { Cuantitativo, } \\
\text { correlacional, } \\
\text { muestra de } 630 \\
\text { profesoras. }\end{array}$ & $\begin{array}{l}\text { Los factores clásicos relacionados al trabajo } \\
\text { apenas contribuyen a la salud mental, } \\
\text { resultando la relación esfuerzo-recompensa el } \\
\text { factor más importante identificado, seguido de } \\
\text { los complejos físicos y factores personales. } \\
\text { Estos factores debieran ser intervenidos a fin } \\
\text { de prevenir consecuencias en la salud mental. }\end{array}$ & 3 \\
\hline E.11 & $\begin{array}{l}\text { Trabalho, } \\
\text { Educação e Saúde/ } \\
\text { Scielo. 2018. Brasil } \\
\text { (Albuquerque et al., } \\
\text { 2018) }\end{array}$ & $\begin{array}{c}\text { Estudio } \\
\text { cuantitativo, } \\
\text { transversal, } \\
\text { participaron } 1201 \\
\text { docentes. }\end{array}$ & $\begin{array}{l}\text { Los factores carga horaria semanal, número de } \\
\text { alumnos por clase y el número de clases por } \\
\text { docente presentaron asociación con trastornos } \\
\text { psiquiátricos menores. Las alteraciones } \\
\text { psíquicas son muy altas entre los docentes, } \\
\text { existiendo indicios de asociación con diversas } \\
\text { formas de explotación en el trabajo docente. }\end{array}$ & 3 \\
\hline
\end{tabular}


Tabla 1. Continuación...

\begin{tabular}{|c|c|c|c|c|}
\hline Código & $\begin{array}{c}\text { Revista/ } \\
\text { Indexación, año y } \\
\text { lugar de ejecución }\end{array}$ & Método y muestra & Principales resultados & $\begin{array}{l}\text { Nivel de } \\
\text { evidencia }\end{array}$ \\
\hline E. 12 & $\begin{array}{l}\text { Health and quality } \\
\text { of life outcomes/ } \\
\text { Web of Science. } \\
2015 . \text { China } \\
\text { (Liu et al., 2015) }\end{array}$ & $\begin{array}{c}\text { Estudio } \\
\text { cuantitativo, } \\
\text { transversal, muestra } \\
965 \text { profesores. }\end{array}$ & $\begin{array}{l}\text { Los profesores experimentaron bajo nivel de } \\
\text { calidad de vida relacionado con salud } \\
\text { (CVRS), viéndose más afectada la salud } \\
\text { mental que la salud física. Por su parte, los } \\
\text { factores del comportamiento organizativo } \\
\text { tales como identificación grupal, percepción } \\
\text { de apoyo organizacional y empoderamiento } \\
\text { psicológico, son predictores de la CVRS y } \\
\text { son recursos positivos para mejorarla. }\end{array}$ & 3 \\
\hline E.13 & $\begin{array}{l}\text { Ciencia y Trabajo/ } \\
\text { Scielo. 2017. Brasil } \\
\text { (Amorim et al., } \\
\text { 2017) }\end{array}$ & $\begin{array}{c}\text { Estudio } \\
\text { cuantitativo, } \\
\text { descriptivo, } \\
\text { transversal, muestra } \\
89 \text { profesores. }\end{array}$ & $\begin{array}{l}\text { La mayoría de los profesores tenían buena } \\
\text { habilidad para el trabajo y un moderado } \\
\text { nivel de fatiga de carácter mental. Mientras } \\
\text { aumenta la presencia de fatiga, disminuye la } \\
\text { habilidad para el trabajo. }\end{array}$ & 4 \\
\hline E. 14 & $\begin{array}{l}\text { Ciencia y Trabajo/ } \\
\text { Scielo. 2018. Chile } \\
\text { (Arteaga } \\
\text { González et al., } \\
\text { 2018). }\end{array}$ & $\begin{array}{c}\text { Estudio } \\
\text { cuantitativo, } \\
\text { descriptivo, } \\
\text { correlacional, } \\
\text { transversal } 156 \\
\text { educadoras. }\end{array}$ & $\begin{array}{l}\text { Existe una relación altamente significativa en la } \\
\text { función fisica con el ausentismo y dependencia } \\
\text { laboral, y significativa con el número de niños a } \\
\text { cargo y ańos laborales. Se suma la alta relación entre } \\
\text { el dolor corporal, los días de ausentismo, la vitalidad } \\
\text { y el número de nińos a cargo, y el rol emocional } \\
\text { con los días de ausentismo laboral, entre otros. La } \\
\text { salud mental se aprecia como buena. }\end{array}$ & 3 \\
\hline E.15 & $\begin{array}{c}\text { Trabalho, } \\
\text { Educação e Saúde/ } \\
\text { Scielo. 2015. Brasil } \\
\text { (Conceição et al., } \\
\text { 2015) } \\
\end{array}$ & $\begin{array}{l}\text { Estudio cuantitativo, } \\
\text { transversal, } \\
\text { correlacional, muestra } \\
175 \text { profesores. }\end{array}$ & $\begin{array}{l}\text { El 19,5\% de los docentes reporta alteraciones } \\
\text { de salud mental, lo cual, era más prevalente en } \\
\text { aquellos profesores que realizan mayor esfuerzo } \\
\text { en el trabajo y menos frecuentes en aquellos con } \\
\text { mejor calidad de vida en dominio físico. }\end{array}$ & 3 \\
\hline E.16 & $\begin{array}{l}\text { Occupational } \\
\text { Medicine/ Web of } \\
\text { Science. } 2015 . \\
\text { Alemania } \\
\text { (Adams et al., } \\
\text { 2015) }\end{array}$ & $\begin{array}{l}\text { Estudio cuantitativo, } \\
\text { transversal, } \\
\text { participaron } \\
\text { profesores y personal } \\
\text { educativo } 13 \text { colegios }\end{array}$ & $\begin{array}{l}\text { 36\% de los docentes fueron clasificados con } \\
\text { patrón de afrontamiento de riesgo relacionado } \\
\text { al trabajo excesivamente ambicioso o } \\
\text { resignado. } 45 \% \text { tenían un patrón ambicioso } \\
\text { saludable. Los factores emocionales y sociales } \\
\text { de profesores y personal educativo estaban } \\
\text { asociados a patrones de afrontamiento } \\
\text { riesgosos y poco ambiciosos, los cuales } \\
\text { deberían ser focos de intervención. }\end{array}$ & 3 \\
\hline E.17 & $\begin{array}{c}\text { Salud de los } \\
\text { trabajadores/ } \\
\text { Scielo. } 2013 \text {. Chile } \\
\text { (Muñoz \& Lucero, } \\
\text { 2013) }\end{array}$ & $\begin{array}{l}\text { Estudio mixto, } \\
\text { donde se utilizó el } \\
\text { método cualitativo } \\
\text { de estudio de caso, } \\
\text { en el que se } \\
\text { entrevistaron } 15 \\
\text { trabajadoras de una } \\
\text { escuela especial. }\end{array}$ & $\begin{array}{l}\text { Se destaca un buen clima laboral y liderazgo } \\
\text { participativo por parte de la dirección de la } \\
\text { escuela, como facilitadores del desarrollo de } \\
\text { una intervención efectiva que permite } \\
\text { mejorar la calidad del trabajo y salud física. } \\
\text { Cerca de la mitad de las trabajadoras } \\
\text { presentaban riesgo en su salud mental y } \\
\text { cansancio emocional, y más del 90\% poseía } \\
\text { baja despersonalización, alta realización } \\
\text { personal y buena calidad de vida global en el } \\
\text { trabajo. En las entrevistas se evidenció } \\
\text { cansancio físico, agresiones de parte de los } \\
\text { escolares, lesiones musculares por fuerza mal } \\
\text { realizada y sensación de existir una carga de } \\
\text { trabajo mal distribuida, lo cual generaba un } \\
\text { malestar encubierto entre las trabajadoras. }\end{array}$ & 5 \\
\hline
\end{tabular}


Tabla 1. Continuación...

\begin{tabular}{|c|c|c|c|c|}
\hline Código & $\begin{array}{c}\text { Revista/ } \\
\text { Indexación, año y } \\
\text { lugar de ejecución }\end{array}$ & Método y muestra & Principales resultados & $\begin{array}{l}\text { Nivel de } \\
\text { evidencia }\end{array}$ \\
\hline E.18 & $\begin{array}{l}\text { Salud Uninorte/ } \\
\text { Scopus. } 2017 . \\
\text { Colombia (Acosta- } \\
\text { Fernández et al., } \\
\text { 2017) }\end{array}$ & $\begin{array}{c}\text { Estudio } \\
\text { cuantitativo, no } \\
\text { experimental, } \\
\text { transversal, } \\
\text { analítico y } \\
\text { explicativo, con } \\
\text { una muestra } 68 \\
\text { docentes } \\
\text { universitarios. }\end{array}$ & $\begin{array}{l}\text { Los docentes refirieron altas exigencias } \\
\text { laborales con condiciones de trabajo de } \\
\text { impacto negativo, expresadas en molestias } \\
\text { como verbalización constante, posturas } \\
\text { incómodas y tareas complejas. Además, se } \\
\text { encontró que ambos grupos desempeñan su } \\
\text { trabajo en condiciones psicosociales } \\
\text { desfavorables. Además, se identificó presencia } \\
\text { de exposición de violencia en } 80,8 \% \text { de los } \\
\text { docentes. Así, } 2 / 3 \text { docentes requerirín de una } \\
\text { intervención inmediata para mejoramiento de } \\
\text { sus condiciones de trabajo. }\end{array}$ & 4 \\
\hline E.19 & $\begin{array}{l}\text { Revista Portuguesa } \\
\text { de Enfermagem de } \\
\text { Saúde Mental/ } \\
\text { Scielo. 2017. Brasil } \\
\text { (Torres et al., } \\
\text { 2017) }\end{array}$ & $\begin{array}{l}\text { Estudio } \\
\text { cuantitativo, } \\
\text { transversal, } \\
\text { exploratorio, } \\
\text { muestra } 525 \\
\text { profesores. }\end{array}$ & $\begin{array}{c}\text { Se encontró una prevalencia de } 37,1 \% \text { de } \\
\text { trastornos mentales comunes. Se constató } \\
\text { que la agresión física y verbal contra el } \\
\text { profesor, la agresión o amenaza con arma de } \\
\text { fuego o arma blanca, el tráfico y el consumo } \\
\text { de drogas en la escuela están asociados a la } \\
\text { presencia de trastornos mentales comunes en } \\
\text { docentes. }\end{array}$ & 3 \\
\hline E. 20 & $\begin{array}{l}\text { Salud Uninorte/ } \\
\text { Scopus. } 2013 . \\
\text { México } \\
\text { (Aldrete et al., } \\
\text { 2013) }\end{array}$ & $\begin{array}{l}\text { Estudio } \\
\text { cuantitativo, } \\
\text { transversal, } \\
\text { analítico en } \\
\text { muestra } 286 \\
\text { profesoras. }\end{array}$ & $\begin{array}{c}\text { El 45,5\% de los participantes presentó } \\
\text { perturbación del sueño y } 43 \% \text { síntomas } \\
\text { somáticos. Se evidenció asociación y riesgo } \\
\text { cuando se percibe que el trabajo tiene que } \\
\text { ver con problemas de salud. Sumado a } \\
\text { ausentismo. }\end{array}$ & 3 \\
\hline
\end{tabular}

Fuente: Elaboración propia.

Posterior a la descripción de los resultados, y siguiendo con el método de Souza et al. (2010), se realizó un análisis crítico de la literatura internacional localizada en la búsqueda, para responder al objetivo de la revisión de la literatura e identificar las tres categorías de argumentación, a saber: 1) Condiciones laborales de los profesores, 2) Efectos del trabajo en la salud de los profesores, 3) Carga mental de trabajo en los profesores, las cuales se resumen en la Tabla 2.

Tabla 2. Clasificación de los artículos según categorías.

\begin{tabular}{cc}
\hline Categorías & Estudios seleccionados \\
\hline Condiciones laborales de los profesores & E.1; E.3; E.5, E.6; E.8; E.9; E.11, E.12; E.18; E.19 \\
\hline Efectos del trabajo en la salud de los profesores & $\begin{array}{c}\text { E.1; E.2; E,4; E.5; E.6; E.7; E.8; E.9; E.10; E.12; E.13; } \\
\text { E.14; E.15; E.16; E.17; E.18; E.19; E.20 }\end{array}$ \\
\hline Carga mental de trabajo en los profesores & E.12; E.13; E.14; E.16; E.17 \\
\hline
\end{tabular}

Fuente: Elaboración propia.

Finalmente, los autores del artículo proponen un apartado de Sugerencias para la salud ocupacional basadas en hallazgos de la revisión, dada la evidencia encontrada. 


\section{Discusión}

\section{Condiciones laborales de los profesores}

En relación a las condiciones laborales de los profesores de establecimientos educacionales primarios y secundarios, los estudios localizados han descrito factores negativos que condicionan el trabajo docente. Entre estos factores negativos, se han identificado características del entorno de trabajo como la infraestructura del lugar y percepción de falta de recursos; condiciones laborales como bajas remuneraciones, gran número de estudiantes por sala, número de clases y de actividades, así como la percepción de inequidad en condiciones, e inseguridad relacionada a la estabilidad laboral, sumado a la intensidad y tiempos largos de trabajo, el ruido en la sala de clases que exige elevar la voz, presencia de corrientes de aire y cambios de temperatura en sala de clases, y exposición a ruido ambiental; sumado a percepciones negativas de las relaciones interpersonales que contemplan problemas con estudiantes, exigencias emocionales, exposición a situaciones de violencia y baja calidad de las relaciones sociales en el trabajo (Acosta-Fernández et al., 2017; Castro \& Muñoz, 2013; Albuquerque et al., 2018; Cezar-Vaz et al., 2015; Guerrero-Barona et al., 2018; Mora Pino et al., 2018; Guerreiro et al., 2016; Sousa \& Barros, 2017; Torres et al., 2017).

En cuanto a los factores positivos se destacan las buenas relaciones con alumnos, colegas y autoridades de los establecimientos, la motivación por el trabajo, la oportunidad para expresar opiniones, la identidad de ser profesor y el reconocimiento del trabajo (Cezar-Vaz et al., 2015; Guerreiro et al., 2016). Además, un estudio en China señaló que ciertos factores del comportamiento organizacional como la identificación grupal, la percepción de apoyo por parte de la organización y el empoderamiento psicológico, son predictores de la calidad de vida relacionada con salud y representan recursos positivos para mejorarla (Liu et al., 2015).

\section{Efectos del trabajo en la salud de los profesores}

Las exigencias que asumen los docentes para cumplir con las responsabilidades ligadas a su trabajo, se han asociado con daños para la salud de acuerdo a los resultados de la revisión bibliográfica. Las consecuencias que surgen a raíz de los intentos por superar las condiciones precarias de trabajo, llevan a una connotación negativa de las cargas fisiológicas, físicas y mentales (Guerreiro et al., 2016). Así, se han descrito efectos en la salud y factores de riesgo tales como síntomas depresivos, estrés relacionado a factores de la escuela, agotamiento, cansancio físico y fatiga generalizada, lesiones musculares, perturbación del sueño y exposición a situaciones y comportamientos de violencia en el contexto laboral educacional (AcostaFernández et al., 2017; Aldrete et al., 2013; Amorim et al., 2017; Cezar-Vaz et al., 2015; Guerrero-Barona et al., 2018; Herman et al., 2020; Muñoz \& Lucero, 2013).

En detalle, desde el punto de vista fisiológico se describen con mayor frecuencia problemas vasculares y várices en miembros inferiores, colon irritable, disfonías o afonías, y desórdenes del dormir (Castro \& Muñoz, 2013; Cezar-Vaz et al., 2015; Silva \& Pinheiro, 2017). Con respecto a la disfonía, un estudio realizado en Chile señala que existen factores personales y laborales-ambientales como la hidratación insuficiente, elevar la intensidad de la voz, gritar y carraspear, y el alto consumo de irritantes laríngeos (cafeína, aliños, bebidas gasificadas); y en cuanto al contexto ocupacional se encontró que el $82 \%$ de los encuestados ocupa la voz por más de 31 horas a la semana en el contexto laboral (Mora Pino et al., 2018). 
Se han identificado actividades que representan mayor demanda física, dentro de las cuales se encuentran la corrección de materiales y actividades en grupo con alumnos, que se pueden traducir en efectos para la salud como dolor de miembros superiores e inferiores, cabeza y columna (Silva \& Pinheiro, 2017).

En cuanto a demandas mentales, la principal causa es el desinterés por parte de alumnos y padres, las cuales se pueden traducir en síntomas emocionales como irritabilidad, desánimo e insomnio, pudiendo favorecer la aparición de estrés, trastornos de ansiedad y depresión (Silva \& Pinheiro, 2017). Un estudio realizado en profesores municipales de Brasil, señaló que existe una alta prevalencia de enfermedades de salud mental en los profesores, lo cual concuerda con lo encontrado en literatura internacional (Baldacara et al., 2015; Carlotto \& Câmara, 2015; Conceição et al., 2015; Muñoz \& Lucero, 2013; Torres et al., 2017). Por su parte, se han pesquisado factores relacionados al aumento en la probabilidad de padecer trastornos mentales comunes, como lo son la ambigüedad del rol, entendida como la poca claridad en las expectativas relacionadas a las tareas del individuo en su quehacer laboral, la sobrecarga mental, bajo nivel de apoyo social y baja percepción de autoeficacia (Carlotto \& Câmara, 2015). Además, se ha evidenciado asociaciones significativas entre ciertas condiciones laborales y efectos en la salud mental, como un salario inadecuado y ansiedad, y entre excesivo número de actividades y riesgos psicosociales como estrés y sobrecarga mental (Cezar-Vaz et al., 2015; Guerrero-Barona et al., 2018; Liu et al., 2015; Seibt et al., 2013).

Por el contrario, los docentes reportaron que una buena relación entre colegas promueve el bienestar en el lugar de trabajo, así como también se destaca la relación entre una alta calidad de vida en el trabajo con: el bienestar psicológico, el balance vidatrabajo y un bajo nivel de trastornos psicológicos (Cezar-Vaz et al., 2015).

Algunos factores y características del trabajo sin duda afectan la calidad laboral y el estado de salud de los trabajadores, por tanto, como se señaló en un estudio realizado en Chile con educadoras de párvulos, se debe potenciar el buen clima laboral y liderazgo participativo por parte de los directivos, como factores facilitadores del desarrollo de una intervención efectiva que permita mejorar estos aspectos (Muñoz \& Lucero, 2013). A su vez, otro estudio de similares características demostró una relación altamente significativa entre la función física con ausentismo y dependencia laboral, una alta relación entre dolor corporal, días de ausentismo, vitalidad y número de nińos a cargo, y rol emocional con días de ausentismo laboral, entre otros factores que resultan importantes de ser intervenidos (Arteaga González et al., 2018).

Cabe destacar que, además de considerarse las condiciones de trabajo perjudiciales para la salud, estas pudieran interferir en el aprendizaje de los estudiantes, poniendo en riesgo el proceso educativo y condicionando el ejercicio saludable de las actividades de trabajo de los docentes (Silva \& Pinheiro, 2017). Con esto, se evidencia una necesidad de formular intervenciones que promuevan espacios laborales saludables para los profesores, que intervengan en los factores críticos, como los emocionales, ambientales, laborales y sociales, y que favorezcan patrones de afrontamiento efectivos (Adams et al., 2015).

\section{Carga mental de trabajo en los profesores}

La revisión de literatura no arrojó resultados de estudios que midieran la carga mental de trabajo percibida en profesores primarios y secundarios, con algún instrumento validado y específico para su valoración, sin embargo, sí se localizan estudios que describen ampliamente algunas características de los entornos laborales y personales del 
docente, y efectos en la salud, que se han relacionado a la percepción de la carga mental de trabajo, lo cual será detallado a continuación.

Dentro de las características del entorno de trabajo y de la tarea, están las demandas cognitivas (Díaz et al., 2012). Para los docentes estas demandas, se ven reflejadas en actividades como la preparación de clases y materiales, corrección de trabajos, evaluaciones, entre otras; sumado a la organización temporal y las condiciones de ambientales de iluminación, sonoridad y temperatura, el grado de centralización en la toma de decisiones y las demandas emocionales (Adams et al., 2015; Arteaga González et al., 2018; Ceballos et al., 2016; Díaz et al., 2012; Rodríguez-Martínez et al., 2018). Por su parte, son características personales del docente relacionadas a la carga mental de trabajo la edad, el sexo, el estado de salud, problemas familiares y características de la personalidad, entre otras (Ceballos-Vásquez et al., 2015; Díaz et al., 2012). En cuanto a los efectos en la salud que se han descrito relacionados a la carga mental se puede destacar el grado de fatiga (Amorim et al., 2017).

A su vez se ha evidenciado que la percepción del entorno laboral y sus factores implicados, tendrá repercusiones y asociaciones significativas en el desempeño del trabajador (Díaz et al., 2012), así como también efectos en la percepción de calidad de vida relacionada con salud (Liu et al., 2015; Muñoz \& Lucero, 2013; Costa et al., 2020).

\section{Sugerencias para la Salud Ocupacional Basadas en Hallazgos de la Revisión}

Tras reconocer que el trabajo docente y sus efectos en la salud contemplan una serie de factores implicados en la percepción de carga mental de trabajo, se hace necesario apuntar a las medidas que permitirán a los trabajadores y las instituciones educativas amortiguar sus efectos y promover entornos saludables de trabajo.

Un importante factor amortiguador en la percepción de riesgos psicosociales en el trabajo es el apoyo social, entendido como el apoyo que percibe el trabajador en el contexto laboral, y que fue descrito por Karasek \& Theorell (1990), destacando que si es percibido de forma positiva el entorno psicosocial pudiera percibirse de mejor manera, relacionándose a mejores niveles de satisfacción laboral, compromiso docente y mejorando el trabajo en equipo (Cezar-Vaz et al., 2015; Extremera et al., 2019; Guerrero-Barona et al., 2018; Karasek \& Theorell, 1990).

En la revisión se destaca la evidencia en cuanto a efectos positivos del trabajo docente, puntos esenciales a promover, entre los que se encuentran las relaciones interpersonales entre los docentes, y los lazos que establecen con sus estudiantes. Esto sin duda retribuye a lo expuesto previamente sobre el apoyo social, pero también tiene un valor emocional significativo, sumado a la identidad docente, con un reconocimiento efectivo, y la motivación por el trabajo. Además, se debe enfatizar el desarrollo propio del trabajador en favor de sus capacidades para el trabajo, lo que permite darle un significado a éste, y aumentar el sentido de pertenencia en él (Katsantonis, 2020; Zamora-Díaz et al., 2017).

Ante esto, estudios han señalado la importancia de generar estrategias de afrontamiento en los docentes, que apunten a evitar el aumento del estrés y la consiguiente enfermedad (Herman et al., 2020; Oliveira et al., 2017).

A partir de la revisión de la literatura, los autores sugieren (Díaz et al., 2012; Ceballos, 2015; Ceballos et al., 2016; Barrios Pérez et al., 2019; Murillo et al., 2020): 
1. Para las instituciones educacionales se sugiere promover el desarrollo de evidencia en materia de salud laboral dirigida a profesionales de la educación, con el fin de sustentar la promoción de ambientes saludables para el trabajo con base empírica;

2. Realizar diagnóstico de los factores psicosociales percibidos por los docentes: específicamente, realizar mediciones de la carga mental de trabajo de los profesores, con instrumentos validados y estandarizados para cada país, que permita abordar los factores que inciden en ella y que pueden afectar la percepción del entorno laboral y la salud del profesor;

3. Coordinar aspectos de organización del trabajo como son los plazos en entrega de actividades docentes comprometidas, planificación de vacaciones o permisos con anterioridad, y fortalecer el nivel de autonomía del puesto docente para disminuir la ambigüedad en la toma de decisiones;

4. Implementación de pausas efectivas entre actividades académicas (vuelta a la calma), al menos cada dos horas, y la revisión de horas de jornada en extensión y carga laboral;

5. Capacitar a los trabajadores de las instituciones educativas en liderazgo y trabajo en equipo para mejorar el apoyo social y la calidad de vida laboral;

6. Desde la salud ocupacional, se debe profundizar su estudio como un polo incipiente para el ejercicio de los profesionales de la salud y la formulación de nuevos conocimientos en esta materia, con el fin de mejorar la salud de los trabajadores y la promoción de ambientes saludables de trabajo, la educación al docente en el autocuidado, manejo del estrés, la prevención de riesgos laborales y la conciliación trabajo-familia;

7. Fortalecer vínculos de trabajo colaborativo entre las entidades sanitarias y de educación, con el fin de formular y/o proponer manuales o protocolos para responder a necesidades tales como: evaluación del puesto de trabajo, uso adecuado de la voz, uso de elementos ergonómicos, controles de salud preventivos al interior de los centros educacionales, entre otros.

\section{Conclusiones y Perspectivas}

Los hallazgos evidenciados en esta revisión permiten a los autores concluir que:

El trabajo docente se ve expuesto a múltiples riesgos psicosociales y factores determinantes de ellos. Se evidenciaron condiciones laborales que representan riesgos para la salud de los docentes, así como algunas características personales que podrían afectar su capacidad de afrontarlos.

Los profesores trabajan con alta intensidad y tiempos muy acotados, requiriendo realizar actividades fuera de horario para responder a las exigencias de su trabajo. Además, deben responder a factores ambientales, trabajar con una alta cantidad de estudiantes y los factores relacionados a ello, se desenvuelven en ambientes de trabajo en el que hay presencia de mala calidad de relaciones interpersonales en los equipos y otros aspectos que se relacionan a la inestabilidad laboral y la falta de recursos para el desarrollo de sus tareas.

Por otra parte, los efectos en la salud de los profesores son ampliamente descritos en la literatura, y coinciden en destacar problemas de salud física y mental, los cuales condicionarían el ejercicio saludable de las actividades de trabajo de los docentes. 
Aun así, se destacan aspectos positivos como la identidad de ser profesor y el reconocimiento del trabajo y, en algunos casos, la buena relación entre colegas, la cual promueve el bienestar en el lugar de trabajo y potencian un adecuado entorno de trabajo.

El estudio de la carga mental de trabajo como factor psicosocial laboral de los profesores representa un desafío para todas las disciplinas que contribuyen a la seguridad y salud en el trabajo. Esto plantea una brecha para la investigación, tanto en generar evidencia en torno a la salud laboral que contribuya a mejorar los entornos de trabajo, como para potenciar las características y habilidades propias del trabajador en favor de su salud.

Es así como surge la necesidad de contribuir al estudio de la carga mental, realizando mediciones objetivas y subjetivas de los factores asociados a ella y al entorno laboral, con el fin de asegurar un trabajo saludable para los docentes, con baja cantidad de riesgos para su salud desde una perspectiva integral, y que, finalmente, permitan intervenir los riesgos psicosociales y contribuir a un adecuado proceso educativo.

\section{Referencias}

Acosta-Fernández, M., Parra-Osorio, L., Restrepo-García, J., Pozos-Radillo, B., Aguilera-Velasco, M., \& Torres-López, T. (2017). Condiciones psicosociales, violencia y salud mental en docentes de medicina y enfermería. Salud Uninorte, 33(3), 344-354.

Adams, J., Dudenhöffer, S., Claus, M., Kimbel, R., Letzel, S., \& Rose, D. (2015). Coping patterns in special school staff: demographic and organizational factors. Occupational Medicine, 66(2), 138-142. http://dx.doi.org/10.1093/occmed/kqv157.

Albuquerque, G. S. C., Lira, L. N. A., Santos Junior, I., Chiochetta, R. L., Perna, P. O., \& Silva, M. J. S. (2018). Exploração e sofrimento mental de professores: um estudo na rede estadual de ensino do Paraná. Trabalho, Educação e Saúde, 16(3), 1287-1300. http://dx.doi.org/10.1590/1981-7746sol00145.

Aldrete, G., León, S., González, R., Medina, E., Contreras, M., \& Pérez, B. (2013). El trabajo y la salud mental de las profesoras de preescolar de la zona metropolitana de Guadalajara. Salud Uninorte, 29(3), 478-486.

Amorim, T., Ferreira, A., \& Gomes, N. (2017). Influência da fadiga ocupacional na capacidade para o trabalho de professores universitários. Ciencia \& Trabajo, 19(59), 86-90. http://dx.doi.org/10.4067/S0718-24492017000200086.

Arteaga González, P., Hermosilla-Ávila, A., Mena Bastías, C., \& Contreras Contreras, S. (2018). Una mirada a la calidad de vida y salud de las educadoras de Párvulos. Ciencia \& Trabajo, 20(61), 42-47. http://dx.doi.org/10.4067/S0718-24492018000100042.

Baldacara, L., Ferreira, A., Gerley, J., \& Carvalho, G. (2015). Common psychiatric symptoms among public school teachers in Palmas, Tocantins, Brazil: an observational cross-sectional study. Sao Paulo Medical Journal, 133(5), 435-438. http://dx.doi.org/10.1590/1516-3180.2014.8242810.

Barrios Pérez, L. S., De La Rosa Barbosa, M. J., Liñán Bernal, S. S., Alvarino Cruz, C., \& Arrazola, M. (2019). Condiciones medioambientales de docentes y trabajadores de la institución educativa Salvador entregas del distrito de Barranquilla, 2018. Enfoque Latinoamericano, 2(2), 51-68. http://dx.doi.org/10.32012/26195399/rel22201960.

Boada-Grau, J., \& Ficapal-Cusí, P. (2012). Salud y trabajo los nuevos y emergentes riesgos psicosociales. Barcelona: Editorial UOC.

Boström, M., Björklund, C., Bergström, G., Nybergh, L., Schäfer Elinder, L., Stigmar, K., Wåhlin, C., Jensen, I., \& Kwak, L. (2019). Health and work environment among female and male swedish elementary school teachers-a cross-sectional study. International Journal of Environmental Research and Public Health, 17(1), 227. http://dx.doi.org/10.3390/ijerph17010227. 
Carlotto, M., \& Câmara, S. G. (2015). Prevalence and risk factors of common mental disorders among teachers. Revista de Psicología del Trabajo y de las Organizaciones, 31(3), 201-206.

http://dx.doi.org/10.1016/j.rpto.2015.04.003.

Castro, C., \& Muñoz, A. (2013). Salud y trabajo de docentes de instituciones educativas distritales de la localidad uno de Bogotá. Avances en Enfermería, 31(2), 30-42.

Ceballos, P. (2015). Seguridad y salud en el trabajo: un polo de desarrollo para enfermería. In P. C. Vásquez, V. Guerra-Guerrero, M. Macaya \& C. C. Gonzalez (Eds.), Abordajes disciplinares: una mirada de enfermería a problemáticas actuales (pp. 119-144). Chile: Ediciones Universidad Católica del Maule.

Ceballos, P., Rolo, G., \& Vilches, V. (2016). Carga mental y su repercusión en los entornos saludables de trabajo. In A. C. R. Quinteros (Eds.), Salud en el trabajo: desafios del presente y futuro (pp. 71-85). Chile: Universidad Católica del Maule.

Ceballos-Vásquez, P., Rolo-González, G., Hernández-Fernaud, E., Cabrera, D., Paravic-Klijn, T., \& Burgos-Moreno, M. (2015). Factores psicosociales y carga mental de trabajo: una realidad percibida por enfermeras/os en Unidades Críticas. Revista Latino-Americana de Enfermagem, 23(2), 315-322.

Cezar-Vaz, M., Alvez, C., Capa, M., Pereira, L., \& Miritz, A. (2015). Mental health of elementary school teachers in Southern Brazil: working conditions and health consequences. The Scientific World Journal, 2015, 1-6. http://dx.doi.org/10.1155/2015/825925.

Conceição, R., Pastore, A., Barbosa, M., Lima, S., Silva, J., \& Lima, A. (2015). Transtorno mental e estressores no trabalho entre professores universitários da área da saúde. Trabalho, Educação e Saúde, 13(1), 135-155.

Costa, E., Martínez, M., \& Reschetti, S. (2020). Quality of life, health and work of elementary school teachers. Acta Paulista de Enfermagem, 34, 1-8. http://dx.doi.org/10.37689/acta-ape/2020AO0286.

Díaz, D., Hernández, E., \& Rolo, G. (2012). Carga mental de trabajo. Espańa: Ed. Síntesis.

Extremera, N., Mérida-López, S., Sánchez-Álvarez, N., Quintana-Orts, C., \& Rey, L. (2019). Un amigo es un tesoro: inteligencia emocional, apoyo social organizacional y engagement docente. Praxis \& Saber, 10(24), 69-92. http://dx.doi.org/10.19053/22160159.v10.n25.2019.10003.

Ferreira, M., \& Ferreira, C. (2014). Carga mental e carga psíquica em profissionais de enfermagem. Revista Portuguesa de Enfermagem de Saúde Mental, (esp. 1), 47-52.

Guerreiro, N. P., Nunes, E. F. P. A., González, A. D., \& Mesas, A. E. (2016). Perfil sociodemográfico, condições e cargas de trabalho de professores da rede estadual de ensino de um município da regiáo sul do Brasil. Trabalho, Educação e Saúde, 14(Supl. 1), 197-217. http://dx.doi.org/10.1590/1981-7746-sol00027.

Guerrero-Barona, E., Gómez, R., Moreno-Manso, J., \& Guerrero-Molina, M. (2018). Psychosocial risk factors, perceived stress and mental health in teachers. Revista Clinica Contemporánea, 9, e2.

Herman, K. C., Prewett, S. L., Eddy, C. L., Savala, A., \& Reinke, W. M. (2020). Profiles of middle school teacher stress and coping: concurrent and prospective correlates. Journal of School Psychology, 78, 54-68. http://dx.doi.org/10.1016/j.jsp.2019.11.003.

Ilaja, B., \& Reyes, C. (2016). Burnout and emotional intelligence in university professors: implications for occupational health. Psicologia desde el Caribe, 33(1), 31-46. http://dx.doi.org/10.14482/psdc.33.1.8081.

Karasek, R., \& Theorell, T. (1990). Healthy work: stress, productivity and the reconstruction of working life. New York: Basic Book.

Katsantonis, I. (2020). Investigation of the impact of school climate and teachers' self-efficacy on job satisfaction: a cross-cultural approach. European Journal of Investigation in Health, Psychology and Education, 10(1), 119-133. http://dx.doi.org/10.3390/ejihpe10010011.

Liu, C., Wang, S., Shen, X., Li, M., \& Wang, L. (2015). The association between organizational behavior factors and health-related quality of life among college teachers: a cross-sectional study. Health and Quality of Life Outcomes, 13(1), 1-12. http://dx.doi.org/10.1186/s12955-015-0287-4.

Mora Pino, K. M., Clavijo Rocha, F. J., Galdames Durán, S. P., Maya Molina, C. C., \& Soto Galleguillos, V. A. (2018). Contexto ocupacional, abuso y mal uso vocal en profesores de la Ciudad de Iquique. Ciencia \& Trabajo, 20(62), 116-120. http://dx.doi.org/10.4067/S0718-24492018000200116. 
Muñoz, M., \& Lucero, B. (2013). Calidad de vida profesional de trabajadoras de una escuela de estudiantes con discapacidades múltiples. Salud de los Trabajadores, 21(2), 151-162.

Murillo, K. D. M., Suárez, O. B. G., \& Moreno-Chaparro, J. (2020). Estrategias de intervención de los factores de riesgo psicosocial de origen laboral: una visión desde terapia ocupacional. Cadernos Brasileiros de Terapia Ocupacional, 28(2), 436-451. http://dx.doi.org/10.4322/2526-8910.ctoAO1934.

Oliveira, L., Azevedo, V., Sampaio, G., Mendonca, V., Feitosa, V., \& Barbosa, I. (2017). Coping strategies used by teachers of the nursing graduation course. Journal of Nursing, 11(11), 4546-4553. http://dx.doi.org/10.1590/0034-7167.2015680503i.

Organización Mundial de la Salud - OMS. (2015). Plan de acción sobre la salud de los trabajadores 20152025. Washington: OMS.

Oxford Centre for Evidence-Based Medicine - CEBM. (2011). The Oxford 2011 levels of evidence. United Kingdom: CEBM.

Peñaherrera, C., \& Soria, J. (2015). Pregunta de investigación y estrategia PICOT. Revista Medicina FCM-UCSG, 19(1), 66-69. http://dx.doi.org/10.23878/medicina.v19i1.647.

Rodríguez-Martínez, M., Tovalin-Ahumada, J. H., Gil-Monte, P. R., Salvador-Cruz, J., \& AcleTomasini, G. (2018). Trabajo emocional y estresores laborales como predictores de ansiedad y depresión en profesores universitarios mexicanos. Informació Psicològica, (115), 93-106. http://dx.doi.org/10.14635/IPSIC.2018.115.11.

Seibt, R., Spitzer, S., Druschke, D., Scheuch, K., \& Hinz, A. (2013). Predictors of mental health in female teachers. International Journal of Occupational Medicine and Environmental Health, 26(6), 856-869. http://dx.doi.org/10.2478/s13382-013-0161-8.

Silva, N., \& Pinheiro, D. M. (2017). Agruras no trabalho do professor. Cadernos Brasileiros de Terapia Ocupacional, 25(4), 713-721. http://dx.doi.org/10.4322/2526-8910.ctoAO0928.

Sousa, D., \& Barros, C. (2017). Ser professor no contexto atual de trabalho: riscos psicossociais e consequências para a saúde e bem-estar. International Journal on Working Conditions, (14), 17-32.

Souza, M., Silva, M., \& Carvalho, R. (2010). Revisão integrativa: o que é e como fazer. Einstein, 8(1), 102-106. http://dx.doi.org/10.1590/s1679-45082010rw1134.

Torres, A., Silva, V., \& Costa, A. (2017). Violência na escola e transtornos mentais comuns em professores. Portuguese Journal of Mental Health Nursing, (18), 31-36.

Zamora-Díaz, W., Cobos-Sanchiz, D., \& López-Noguero, F. (2017). Condiciones sociales y salud laboral del profesorado nicaragüense de secundaria. Revista de Pedagogía, 38(103), 192-208.

\section{Contribución de los Autores}

Yanni Lonnette González-Palacios concepción y diseño del texto, adquisición de datos, análisis e interpretación de datos, y redacción del texto. Paula Andrea CeballosVásquez concepción y diseño del texto, adquisición de datos, análisis e interpretación de datos, redacción del texto y revisión crítica del texto. Flérida Rivera Rojas concepción y diseño del texto, y revisión crítica del texto. Todos los autores aproban la version final del texto.

\section{Autor para la correspondencia}

Paula Andrea Ceballos-Vásquez

e-mail: pceballos@ucm.cl

\section{Editor de sección}

Profa. Dra. Isabela Aparecida de Oliveira Lussi 
Errata

\section{ERRATA: Carga mental en profesores y consecuencias en su salud: una revisión integrativa}

Yanni Lonnette González-Palacios ${ }^{a}$ (D), Paula Andrea Ceballos-Vásquez ${ }^{a}$ (D) , Flérida Rivera-Rojas ${ }^{a}$ (D) ${ }^{a}$ Facultad de Ciencias de la Salud, Universidad Católica del Maule - UCM, Talca, Chile.

Por problemas técnicos durante la producción editorial el artículo "Carga mental en profesores y consecuencias en su salud: una revisión integrativa”, DOI https://doi.org/10.1590/2526-8910.ctoAR2123, publicado en Cadernos Brasileiros de Terapia Ocupacional, 29, e2808, 2021, se publicó con un error.

En la página 1, donde se lee el texto:

https://doi.org/10.1590/2526-8910.ctoAR2123

Debería leer:

https://doi.org/10.1590/2526-8910.ctoAR21232808 